\title{
Kopf-Hals-Tumoren: Profitieren auch ältere Patienten von der Radiochemotherapie?
}

\author{
In einer Studie wurde die Radio- \\ chemotherapie bei älteren Patienten \\ mit Kopf-Hals-Plattenepithelkarzino- \\ men mit der alleinigen Bestrahlung \\ verglichen.
}

\footnotetext{
E twa jeder 4. Patient mit Kopf-Hals- Plattenepithelkarzinom ist $>70$ Jahre alt. Komorbiditäten können hier eine optimale multimodale Therapie erschweren. In einer Auswertung der amerikanischen National Cancer Data Base wurde untersucht, ob ältere Patienten im Hinblick auf das Gesamtüberleben (OS) von einer Radiochemotherapie (RCT) im Vergleich zur alleinigen Bestrahlung profitieren.

Von 4.042 Patienten im Alter von $>70$ Jahren mit nicht metastasierten Tumoren des Oropharynx, Larynx oder Hypopharynx (T3-4 oder $\mathrm{N}+$ ) hatten $63 \%$
}

eine RCT erhalten, $37 \%$ eine alleinige Bestrahlung.

Nach medianem Follow-up von 19 Monaten war die nicht adjustierte Rate für das 2-Jahres-OS in der RCT-Gruppe signifikant höher als nach alleiniger Bestrahlung ( 55,0 vs. $35,1 \%$; $p<0,001)$, ebenso die Rate für das 5-Jahres-OS (30,3 vs. $15,2 \% ; \mathrm{p}<0,001)$. Auch in den multivariaten Analysen und nach Propensity Score Matching zeigte sich ein besseres OS nach RCT als nach alleiniger Bestrahlung. (Hazard Ratio [HR] 0,$63 ; \mathrm{p}<0,001$ bzw. HR 0,73; $\mathrm{p}<0,001$ ). Höheres Alter, zunehmende Komorbiditäten, Larynx- und Pharynxkarzinome sowie höhere $\mathrm{T}$ - und $\mathrm{N}$-Stadien waren mit einem geringeren OS assoziiert.

Eine rekursive Partitionsanalyse ergab, dass die RCT bei Patienten mit einem längeren OS assoziiert war, die $\leq 81$ Jahre alt waren, niedrige Komorbi- ditätsscores nach Charlson-Deyo (CD) aufwiesen und deren Tumor entweder als T1-2/N2-3 oder T3-4/N0-3 klassifiziert wurde. Bei Patienten im Alter von 71-81 Jahren verschwand dieser Vorteil jedoch in zwei Subgruppen: zum einen bei Patienten mit Tumorstadium T1-2/N1 und CD-Score $0-1$ und zum anderen bei Patienten mit Tumorstadium T3-4/N1+ und CD-Score 1+. Bei Patienten im Alter von $>81$ Jahren führte die RCT nicht zu einem längeren Überleben.

Fazit: > 70-jährigen Patienten sollte die RCT zur Behandlung eines Kopf-HalsPlattenepithelkarzinoms nicht wegen des Alters vorenthalten werden. Vielmehr sollten zusätzliche Faktoren wie der Performancestatus und das Tumorstadium mit berücksichtigt werden.

Judith Neumaier

Amini A et al. Survival Outcomes With Concurrent Chemoradiation for Elderly Patients With Locally Advanced Head and Neck Cancer According to the National Cancer Data Base. Cancer. 2016;122(10):1533-4.

\section{Kopf-Hals-Tumoren: Langzeitdaten zum Larynxerhalt}

Bei Patienten mit Larynx- oder Hypopharynxkarzinom kann die Induktionschemotherapie mit Cisplatin, 5-Fluorouracil (5-FU) und Docetaxel die Chance auf Organerhalt verbessern. Nun liegen erstmals 10-Jahres-Daten hierzu vor.

In der Studie GORTEC 2000-01 untersuchte die französische Gruppe für Kopf- und Hals-Tumoren, ob die zusätzliche Gabe von Docetaxel zu einer Induktionschemotherapie mit Cisplatin und 5-FU die Chance auf Larynxerhalt bei Patienten mit Larynx- oder Hypopharynxkarzinomen verbessert. Die Ergebnisse wurden 2009 publiziert. Nun folgten die Langzeitergebnisse über 5 bzw. 10 Jahre.

Untersucht wurden 213 operable, zuvor unbehandelte Patienten mit invasivem Plattenepithelkarzinom (Stadium III oder IV) des Larynx oder Hypopharynx, bei denen eine totale Laryngektomie notwendig erschien. Die Patienten erhielten randomisiert eine Induktionschemotherapie mit Cisplatin plus 5-FU mit oder ohne Docetaxel (3 Zyklen) und, wenn sie ansprachen, anschließend eine Bestrahlung. Der primäre Endpunkt war der 3-Jahres-Erhalt des Larynx. Die mediane Beobachtungsdauer betrug 105 Monate.

Nach der Induktionschemotherapie betrug die Gesamtansprechrate unter Cisplatin, 5-FU und Docetaxel (TPF) 80,0\% und unter Cisplatin und 5-FU alleine (PF) 59,2\% ( $\mathrm{p}=0$ 0,002). Die 5-Jahres-Rate für den Larynxerhalt lag bei 74,0 versus $58,1 \%$, die 10 -Jahres-Rate bei 70,3 versus $46,5 \%(p=0,01)$. Die 5-Jahres-Rate für Überleben ohne Larynxdysfunktion betrug 67,2 gegenüber $46,5 \%$, die 10-Jahres-Rate 63,7 gegenüber 37,2\% $(\mathrm{p}=0,001)$. Gesamtüberleben, krankheitsfreies Überleben und lokoregionäre
Kontrollrate waren unter TPF im Vergleich zu PF nicht verbessert. Späte Toxizitäten vom Grad 3/4 waren unter TPF seltener als unter PF (9,3 vs. $17,1 \%$; $\mathrm{p}=0,038$ ).

Fazit: Auch in der Langzeituntersuchung bestätigte sich, dass die Induktionschemotherapie mit TPF im Vergleich zur Therapie mit PF allein die Chance auf die Erhaltung des Larynx verbessert und die Zeit ohne Larynxdysfunktion verlängert. Daher sollten zur Behandlung von Plattenepithelkarzinomen des Larynx oder Hypopharynx mit dem Ziel des Larynxerhalts eine Chemotherapie mit TPF und eine anschließende Bestrahlung empfohlen werden.

Judith Neumaier

Janoray G et al. Long-Term Results of a Multicenter Randomized Phase III Trial of Induction Chemotherapy With Cisplatin, 5-fluorouracil, \pm Docetaxel for Larynx Preservation. J Natl Cancer Inst. 2016;108(4):djv368. 